\title{
Some New Results on Distance $k$-Domination in Graphs
}

\author{
Samir K. Vaidya ${ }^{1}$ and Nirang J. Kothari ${ }^{2}$ \\ ${ }^{1}$ Department of Mathematics, Saurashtra University, Rajkot, Gujarat 360005, India \\ ${ }^{2}$ B. H. Gardi College of Engineering \& Technology, P.O. Box 215, Rajkot, Gujarat 360001, India
}

Correspondence should be addressed to Samir K. Vaidya; samirkvaidya@yahoo.co.in

Received 17 August 2013; Revised 9 December 2013; Accepted 11 December 2013

Academic Editor: Toufik Mansour

Copyright (C) 2013 S. K. Vaidya and N. J. Kothari. This is an open access article distributed under the Creative Commons Attribution License, which permits unrestricted use, distribution, and reproduction in any medium, provided the original work is properly cited.

We determine the distance $k$-domination number for the total graph, shadow graph, and middle graph of path $P_{n}$.

\section{Introduction}

We begin with finite, connected, and undirected graphs, $G=$ $(V(G), E(G))$ without loops or multiple edges. A dominating set $D$ of a graph $G$ is a set of vertices of $G$ such that every vertex of $V(G)-D$ is adjacent to some vertex of $D$. The domination number $\gamma(G)$ is the minimum cardinality of a dominating set of $G$. Further, the open neighbourhood of $v \in V(G)$ is the set $N(v)=\{u \in V(G) / u v \in E(G)\}$. The closed neighbourhood $N[v]$ of $v \in V(G)$ is the set $N[v]=N(v) \cup\{v\}$. The distance $d(u, v)$ between two vertices $u$ and $v$ is the length of shortest path between $u$ and $v$ in $G$, if exists otherwise, $d(u, v)=\infty$. The open $k$-neighbourhood set $N_{k}(v)$ of vertex $v \in V(G)$ is the set of all vertices of $G$ which are different from $v$ and at distance at most $k$ from $v$ in $G$, that is, $N_{k}(v)=\{u \in$ $V(G) / d(u, v) \leq k\}$. The closed $k$-neighbourhood set $N_{k}[v]$ of $v$ is defined as $N_{k}[v]=N_{k}(v) \cup\{v\}$. Obviously $N(v)=N_{1}(v)$.

The total graph $T(G)$ of a graph $G$ is the graph whose vertex set is $V(G) \cup E(G)$ and two vertices are adjacent whenever they are either adjacent or incident in $G$.

The Shadow graph $D_{2}(G)$ of a connected graph $G$ is obtained by taking two copies of $G$, say $G^{\prime}$ and $G^{\prime \prime}$. Join each vertex $u^{\prime}$ in $G^{\prime}$ to the neighbours of corresponding vertex $u^{\prime \prime}$ in $G^{\prime \prime}$.

The middle graph $M(G)$ of a graph $G$ is the graph whose vertex set is $V(G) \cup E(G)$ and in which two vertices are adjacent whenever either they are adjacent edges of $G$ or one is a vertex of $G$ and the other is an edge incident with it.

For standard terminology and notations we rely upon Balakrishnan and Ranganathan [1] and Haynes et al. [2].
The concept of distance dominating set was initiated by Slater [3] while the term distance $k$-dominating set was coined by Henning et al. [4]. For an integer $k \geq 1$, a $D \subseteq$ $V(G)$ is a $k$-dominating set of $G$ if every vertex in $V(G)-$ $D$ is within distance $k$ from some vertex $v \in D$. That is, $N_{k}[D]=V(G)$. The minimum cardinality among all $k$-dominating sets of $G$ is called the $k$-domination number of $G$ and it is denoted by $\gamma_{k}(G)$. It is obvious that $\gamma(G)=\gamma_{1}(G)$. A $k$-dominating set of cardinality $\gamma_{k}(G)$ is called a $\gamma_{k}$-set. The distance domination in the context of spanning tree is discussed by Griggs and Hutchinson [5] while bounds on the distance two-domination number and the classes of graphs attaining these bounds are reported in the work of Sridharan et al. [6]. In [7] Topp and Volkmann have discussed distance $k$-domination as $k$-covering and characterized connected graphs of order $(k+1) n$ with distance $k$-domination $(k$ covering). Application of distance domination in Ad Hoc wireless networking is briefly discussed by $\mathrm{Wu}$ and $\mathrm{Li}$ [8]. More details and bibliographic references on distance $k$ domination can be found in a survey paper by Henning [9]

\section{Some Definitions and Main Results}

Proposition 1 (see [9]). For $k \geq 1$, let $D$ be a $k$-dominating set of a graph $G$. Then $D$ is a minimal $k$-dominating set of $G$ if and only if each $d \in D$ has at least one of the following properties.

(1) There exists a vertex $v \in V(G)-D$ such that $N_{k}(v) \cap D=$ $\{d\}$.

(2) The vertex $d$ is at distance at least $k+1$ from every other vertex $d$ of $D$ in $G$. 
Theorem 2. For $n \leq 2 k, \gamma_{k}\left(T\left(P_{n}\right)\right)=1$.

Proof. Let $v_{1}, v_{2}, \ldots, v_{n}$ and $e_{1}, e_{2}, \ldots e_{n-1}$ be the vertices and the edges of $P_{n}$, respectively. Then $v_{1}, v_{2}, \ldots, v_{n}, e_{1}, e_{2}, \ldots, e_{n-1}$ will be the vertices of $T\left(P_{n}\right)$. Then $D=\left\{v_{\lfloor n / 2\rfloor}\right\}$ is distance $k$ dominating set of $T\left(P_{n}\right)$ as $n \leq 2 k$. The set $D$ being a singleton set it is obviously a minimal distance $k$-dominating set of $T\left(P_{n}\right)$.

Theorem 3. For $n>2 k$,

$$
\begin{aligned}
& \gamma_{k}\left(T\left(P_{n}\right)\right) \\
& = \begin{cases}\left\lfloor\frac{n}{2 k}\right\rfloor & \text { for } n \equiv 0,1(\bmod 2 k) \\
\left\lfloor\frac{n}{2 k}\right\rfloor+1 & \text { for } n \equiv 2,3, \ldots, 2 k-1(\bmod 2 k)\end{cases}
\end{aligned}
$$

Proof. Let $v_{1}, v_{2}, \ldots, v_{n}$ and $e_{1}, e_{2}, \ldots, e_{n-1}$ be the vertices and the edges of $P_{n}$ respectively. Then $v_{1}, v_{2}, \ldots, v_{n}, e_{1}, e_{2}, \ldots, e_{n-1}$ will be the vertices of $T\left(P_{n}\right)$. Now every vertex from $v_{k+1}$, $v_{k+2}, \ldots, v_{n-k}$ dominates $2 k+1$ vertices of $v_{i}$ 's and $2 k$ vertices of $e_{i}$ 's at a distance $k$ while every vertex from $e_{k}, e_{k+1}, \ldots, e_{n-k}$ dominates $2 k$ vertices of $v_{i}$ 's and $2 k+1$ vertices of $e_{i}$ 's at a distance $k$. Therefore at least one vertex from $\left\{v_{1+(2 k) j}, v_{2+(2 k) j}\right.$, $\left.\ldots, v_{(k+1)+(2 k) j}, v_{(k+2)+(2 k) j}, \ldots, v_{(2 k)+(2 k) j}\right\}$ must belongs to any distance $k$ dominating set $D$ of $T\left(P_{n}\right)$.

Hence,

$$
\gamma_{k}\left(T\left(P_{n}\right)\right) \geq\left\lfloor\frac{n}{2 k}\right\rfloor
$$

Now depending upon the number of vertices of $P_{n}$, consider the following subsets.

For $n \equiv 0,1(\bmod (2 k))$,

$$
D=\left\{v_{(k+1)+(2 k) j} \mid 0 \leq j<\left\lfloor\frac{n}{2 k}\right\rfloor\right\}, \quad|D|=\left\lfloor\frac{n}{2 k}\right\rfloor,
$$

for $n \equiv 2,3, \ldots, k(\bmod (2 k))$,

$$
D=\left\{v_{(k+1)+(2 k) j}, v_{n} \mid 0 \leq j<\left\lfloor\frac{n}{2 k}\right\rfloor\right\}, \quad|D|=\left\lfloor\frac{n}{2 k}\right\rfloor+1,
$$

for $n \equiv k+1, k+2, \ldots, 2 k-1(\bmod (2 k))$,

$$
D=\left\{v_{(k+1)+(2 k) j} \mid 0 \leq j \leq\left\lfloor\frac{n}{2 k}\right\rfloor\right\}, \quad|D|=\left\lfloor\frac{n}{2 k}\right\rfloor+1 .
$$

We claim that each $D$ is a distance $k$ dominating set because

$$
\begin{aligned}
& d\left(v_{(k+1)+(2 k) j}, v_{i+(2 k) j}\right) \leq k, \\
& d\left(v_{(k+1)+(2 k) j}, e_{i+(2 k) j}\right) \leq k,
\end{aligned}
$$

where $1 \leq i \leq 2 k, d\left(v_{n}, v_{n-l}\right) \leq k$, and $d\left(v_{n}, e_{n-l}\right) \leq k$, where, $1 \leq l \leq k$.
Therefore

$$
\begin{array}{r}
N_{k}\left(v_{(k+1)+(2 k) j}\right)=\left\{v_{1+(2 k) j}, v_{2+(2 k) j}, \ldots, v_{k+(2 k) j},\right. \\
v_{(k+2)+(2 k) j}, \ldots, v_{(2 k)+(2 k) j}, \\
e_{1+(2 k) j}, e_{2+(2 k) j}, \ldots e_{k+(2 k) j}, \\
\left.e_{(k+1)+(2 k) j}, \ldots e_{(2 k)+(2 k) j}\right\}, \\
N_{k}\left(v_{n}\right)=\left\{v_{n-1}, v_{n-2}, \ldots, v_{n-k}, e_{n-1}, e_{n-2}, \ldots, e_{n-k}\right\} .
\end{array}
$$

This implies that $N_{k}[D]=V\left(T\left(P_{n}\right)\right)$ for $n \equiv 1,2, \ldots, 2 k(\bmod$ $(2 k))$. Now from the nature of $T\left(P_{n}\right)$, one can observe that every vertex $d$ of $D$ is at a distance at least $k+1$ apart from every other vertex of $D$ in $T\left(P_{n}\right)$.

Thus by Proposition 1 , above defined $D$ is a minimal distance $k$-dominating set of $T\left(P_{n}\right)$. Hence, from (2), for $n>$ $2 k$,

$$
\begin{aligned}
\gamma_{k}\left(T\left(P_{n}\right)\right) & \begin{cases}\left\lfloor\frac{n}{2 k}\right\rfloor & \text { for } n \equiv 0,1(\bmod 2 k) \\
\left\lfloor\frac{n}{2 k}\right\rfloor+1 & \text { for } n \equiv 2,3, \ldots, 2 k-1(\bmod 2 k) .\end{cases}
\end{aligned}
$$

Theorem 4. For $n \leq 2 k+1, \gamma_{k}\left(D_{2}\left(P_{n}\right)\right)=1$.

Proof. Consider two copies of path $P_{n}$. Let $v_{1}, v_{2}, \ldots, v_{n}$ be the vertices of first copy of path $P_{n}$ and $u_{1}, u_{2}, \ldots, u_{n}$ be the vertices of second copy of path $P_{n}$. Then $D=\left\{v_{\lfloor n / 2\rfloor}\right\}$ is distance $k$-dominating set as $n \leq 2 k+1$. The set $D$ being a singleton, set it is obviously a minimal distance $k$-dominating set of $D_{2}\left(P_{n}\right)$.

Theorem 5. For $n>2 k+1$,

$$
\begin{aligned}
\gamma_{k}\left(D_{2}\left(P_{n}\right)\right) & \begin{cases}\left\lfloor\frac{n}{2 k+1}\right\rfloor+1 & \text { for } n \equiv 1,2, \ldots, 2 k(\bmod (2 k+1)) \\
\frac{n}{2 k+1} & \text { for } n \equiv 0(\bmod (2 k+1)) .\end{cases}
\end{aligned}
$$

Proof. Consider two copies of path $P_{n}$. Let $v_{1}, v_{2}, \ldots, v_{n}$ be the vertices of first copy of path $P_{n}$ and $u_{1}, u_{2}, \ldots, u_{n}$ be the vertices of second copy of path $P_{n}$. Now every vertex from $v_{k+1}, v_{k+2}, \ldots, v_{n-k}$ dominates $2 k+1$ vertices of $v_{i}$ 's and $2 k+1$ vertices of $u_{i}$ 's at a distance $k$ while every vertex from $u_{k+1}, u_{k+2}, \ldots, u_{n-k}$ dominates $2 k+1$ vertices of $v_{i}$ 's and $2 k+1$ vertices of $u_{i}$ 's at a distance $k$. Therefore at least one vertex from $\left\{v_{1+(2 k+1) j}, v_{2+(2 k+1) j}, \ldots, v_{(k+1)+(2 k+1) j}, v_{(k+2)+(2 k+1) j}, \ldots\right.$, $\left.v_{(2 k+1)+(2 k+1) j}\right\}$ must belongs to any distance $k$-dominating set $D$ of $D_{2}\left(P_{n}\right)$.

Hence

$$
\gamma_{k}\left(D_{2}\left(P_{n}\right)\right) \geq\left\lfloor\frac{n}{2 k+1}\right\rfloor .
$$

Now depending upon the number of vertices of $P_{n}$, consider the following subsets. 
For $n \equiv 1,2, \ldots, k(\bmod (2 k+1))$,

$$
\begin{array}{r}
D=\left\{v_{(k+1)+(2 k+1) j}, v_{n} \mid 0 \leq j<\left\lfloor\frac{n}{2 k+1}\right\rfloor\right\}, \\
|D|=\left\lfloor\frac{n}{2 k+1}\right\rfloor+1,
\end{array}
$$

for $n \equiv k+1, k+2, \ldots, 2 k(\bmod (2 k+1))$,

$$
\begin{array}{r}
D=\left\{v_{(k+1)+(2 k+1) j} \mid 0 \leq j \leq\left\lfloor\frac{n}{2 k+1}\right\rfloor\right\}, \\
|D|=\left\lfloor\frac{n}{2 k+1}\right\rfloor+1,
\end{array}
$$

for $n \equiv 0(\bmod (2 k+1))$,

$$
\begin{array}{r}
D=\left\{v_{(k+1)+(2 k+1) j} \mid 0 \leq j<\frac{n}{2 k+1}\right\}, \\
|D|=\frac{n}{2 k+1} .
\end{array}
$$

We claim that each $D$ is a distance $k$-dominating set because

$$
\begin{aligned}
& d\left(v_{(k+1)+(2 k+1) j}, v_{i+(2 k+1) j}\right) \leq k, \\
& d\left(v_{(k+1)+(2 k+1) j}, u_{i+(2 k+1) j}\right) \leq k,
\end{aligned}
$$

where $1 \leq i \leq 2 k+1, d\left(v_{n}, v_{n-l}\right) \leq k$ and $d\left(v_{n}, u_{n-l}\right) \leq k$, where $1 \leq l \leq k$.

Therefore

$$
\begin{aligned}
N_{k}\left(v_{(k+1)+(2 k+1) j}\right) & \\
= & \left\{v_{1+(2 k+1) j}, v_{2+(2 k+1) j}, \ldots, v_{k+(2 k+1) j},\right. \\
& v_{(k+2)+(2 k+1) j}, \ldots, v_{(2 k+1)+(2 k+1) j}, \\
& u_{1+(2 k+1) j}, u_{2+(2 k+1) j}, \ldots u_{k+(2 k+1) j}, \\
& \left.u_{(k+1)+(2 k+1) j}, \ldots u_{(2 k+1)+(2 k+1) j}\right\}, \\
N_{k}\left(v_{n}\right)= & \left\{v_{n-1}, v_{n-2}, \ldots, v_{n-k}, u_{n}, u_{n-1}, \ldots, u_{n-k}\right\},
\end{aligned}
$$

which implies that $N_{k}[D]=V\left(D_{2}\left(P_{n}\right)\right)$ for any $n$. Now from the nature of $D_{2}\left(P_{n}\right)$, one can observe that every vertex $d$ of $D$ is at a distance at least $k+1$ apart from every other vertex of $\mathrm{D}$ in $D_{2}\left(P_{n}\right)$.

Thus by Proposition 1 , above defined $D$ is a minimal distance $k$-dominating set of $D_{2}\left(P_{n}\right)$. Hence from (10), for $n>$ $2 k+1$,

$$
\begin{aligned}
\gamma_{k}\left(D_{2}\left(P_{n}\right)\right) \\
= \begin{cases}\left\lfloor\frac{n}{2 k+1}\right\rfloor+1 & \text { for } n \equiv 1,2, \ldots, 2 k(\bmod (2 k+1)) \\
\frac{n}{2 k+1} & \text { for } n \equiv 0(\bmod (2 k+1))\end{cases}
\end{aligned}
$$

Theorem 6. For $n \leq 2 k, \gamma_{k}\left(M\left(P_{n}\right)\right)=1$.
Proof. Let $v_{1}, v_{2}, \ldots, v_{n}$ and $e_{1}, e_{2}, \ldots e_{n-1}$ be the vertices and the edges of $P_{n}$, respectively. Then $v_{1}, v_{2}, \ldots, v_{n}, e_{1}, e_{2}, \ldots, e_{n-1}$ will be the vertices of $M\left(P_{n}\right)$. Then $D=\left\{v_{\lfloor n / 2\rfloor}\right\}$ is distance $k$-dominating set of $M\left(P_{n}\right)$ as $n \leq 2 k$. The set $D$ being a singleton set, it is obviously a minimal distance $k$-dominating set of $M\left(P_{n}\right)$.

Theorem 7. For $n>2 k$,

$$
\begin{aligned}
\gamma_{k}\left(M\left(P_{n}\right)\right) & \begin{cases}\left\lfloor\frac{n}{2 k}\right\rfloor+1 & \text { for } n \equiv 1,2, \ldots, 2 k-1(\bmod (2 k)) \\
\frac{n}{2 k} & \text { for } n \equiv 0(\bmod (2 k)) .\end{cases}
\end{aligned}
$$

Proof. Let $v_{1}, v_{2}, \ldots, v_{n}$ and $e_{1}, e_{2}, \ldots e_{n-1}$ be the vertices and the edges of $P_{n}$ respectively. Then $v_{1}, v_{2}, \ldots, v_{n}, e_{1}, e_{2}, \ldots, e_{n-1}$ will be the vertices of $M\left(P_{n}\right)$. Now every vertex from $v_{k}, v_{k+1}, \ldots, v_{n-k}$ dominates $2 k-1$ vertices of $v_{i}$ 's and $2 k$ vertices of $e_{i}$ 's at a distance $k$ while every vertex from $e_{k}, e_{k+1}, \ldots, e_{n-k}$ dominates $2 k$ vertices of $v_{i}$ 's and $2 k+1$ vertices of $e_{i}$ 's at a distance $k$. Therefore at least one vertex from $\left\{e_{1+(2 k) j}, e_{2+(2 k) j}, \ldots, e_{k+(2 k) j}, e_{k+1+(2 k) j}, \ldots, e_{2 k+(2 k) j}\right\}$ must belong to any distance $k$-dominating set $D$ of $M\left(P_{n}\right)$.

Hence,

$$
\gamma_{k}\left(M\left(P_{n}\right)\right) \geq\left\lfloor\frac{n}{2 k}\right\rfloor
$$

Now depending upon the number of vertices of $P_{n}$, consider the following subsets.

For $n \equiv 1,2, \ldots, k(\bmod (2 k))$,

$$
\begin{array}{r}
D=\left\{e_{k+(2 k) j}, e_{n-1} \mid 0 \leq j<\left\lfloor\frac{n}{2 k}\right\rfloor\right\}, \\
|D|=\left\lfloor\frac{n}{2 k}\right\rfloor+1,
\end{array}
$$

for $n \equiv k+1, k+2, \ldots, 2 k-1(\bmod (2 k))$,

$$
\begin{array}{r}
D=\left\{e_{k+(2 k) j} \mid 0 \leq j \leq\left\lfloor\frac{n}{2 k}\right\rfloor\right\}, \\
|D|=\left\lfloor\frac{n}{2 k}\right\rfloor+1,
\end{array}
$$

for $n \equiv 0(\bmod (2 k))$,

$$
\begin{array}{r}
D=\left\{e_{k+(2 k) j} \mid 0 \leq j<\frac{n}{2 k}\right\}, \\
|D|=\frac{n}{2 k} .
\end{array}
$$

We claim that each $D$ is a distance $k$-dominating set because

$$
\begin{aligned}
& d\left(e_{k+(2 k) j}, e_{i+(2 k) j}\right) \leq k, \\
& d\left(e_{k+(2 k) j}, v_{i+(2 k) j}\right) \leq k,
\end{aligned}
$$

where $1 \leq i \leq 2 k, d\left(e_{n-1}, e_{n-l}\right) \leq k$, and $d\left(e_{n-l}, v_{n-l}\right) \leq k$, where $1 \leq l \leq k$. 
Therefore

$$
\begin{gathered}
N_{k}\left(e_{k+(2 k) j}\right) \\
=\left\{e_{1+(2 k) j}, e_{2+(2 k) j}, \ldots, e_{(k-1)+(2 k) j},\right. \\
e_{(k+1)+(2 k) j}, \ldots, e_{(2 k)+(2 k) j}, \\
v_{1+(2 k) j}, v_{2+(2 k) j}, \ldots v_{k+(2 k) j}, \\
\left.v_{(k+1)+(2 k) j}, \ldots v_{(2 k)+(2 k) j}\right\}, \\
N_{k}\left(e_{n-1}\right)=\left\{e_{n-2}, e_{n-3}, \ldots, e_{n-k}, v_{n-1}, v_{n-2}, \ldots, v_{n-k}\right\} .
\end{gathered}
$$

This implies that $N_{k}[D]=V\left(M\left(P_{n}\right)\right)$ for $n>2 k$. Now from the nature of $M\left(P_{n}\right)$, one can observe that every vertex $d$ of $D$ is at a distance at least $k+1$ apart from every other vertex of $D$ in $M\left(P_{n}\right)$.

Thus by Proposition 1 , above defined $D$ is a minimal distance $k$-dominating set of $M\left(P_{n}\right)$. Hence from (18), for $n>2 k$,

$$
\begin{aligned}
\gamma_{k}\left(M\left(P_{n}\right)\right) \\
= \begin{cases}\left\lfloor\frac{n}{2 k}\right\rfloor+1 & \text { for } n \equiv 1,2, \ldots, 2 k-1(\bmod (2 k)) \\
\frac{n}{2 k} & \text { for } n \equiv 0(\bmod (2 k)) .\end{cases}
\end{aligned}
$$

\section{References}

[1] R. Balakrishnan and K. Ranganathan, A Textbook of Graph Theory, Springer, 2nd edition, 2012.

[2] T. W. Haynes, S. T. Hedetniemi, and P. J. Slater, Fundamentals of Domination in Graphs, Marcel Dekker, New York, NY, USA, 1998.

[3] P. J. Slater, "R-domination in graphs," Journal of Association for Computer Machinery, vol. 23, no. 3, pp. 446-450, 1976.

[4] M. A. Henning, O. R. Swart, and H. C. Swart, "Bounds on distance domination parameters," Journal of Combinatorics, Information and System Sciences, vol. 16, pp. 11-18, 1991.

[5] J. R. Griggs and J. P. Hutchinson, "On the $r$-domination number of a graph,” Discrete Mathematics, vol. 101, pp. 65-72, 1992.

[6] N. Sridharan, V.S. A. Subramanian, and M. D. Elias, "Bounds on the distance two-domination number of a graph," Graphs and Combinatorics, vol. 18, no. 3, pp. 667-675, 2002.

[7] J. Topp and L. Volkmann, "On packing and covering numbers of graphs," Discrete Mathematics, vol. 96, no. 3, pp. 229-238, 1991.

[8] J. Wu and H. Li, "Domination and its application in Ad Hoc wireless networks with unidirectional links," in Proceedings of the 2000 International Conference on Parallel Processing, pp. 189-197, 2000.

[9] M. A. Henning, "Distance domination in graphs," in Domination in Graphs: Advanced, Topics, T. W. Haynes, S. T. Hedetniemi, and P. J. Slater, Eds., pp. 321-349, Marcel Dekker, New York, NY, USA, 1998. 


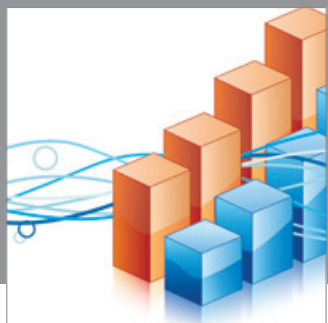

Advances in

Operations Research

mansans

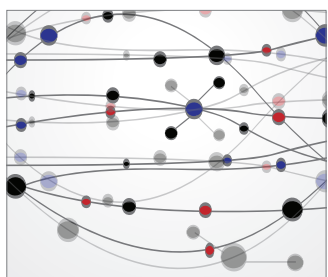

The Scientific World Journal

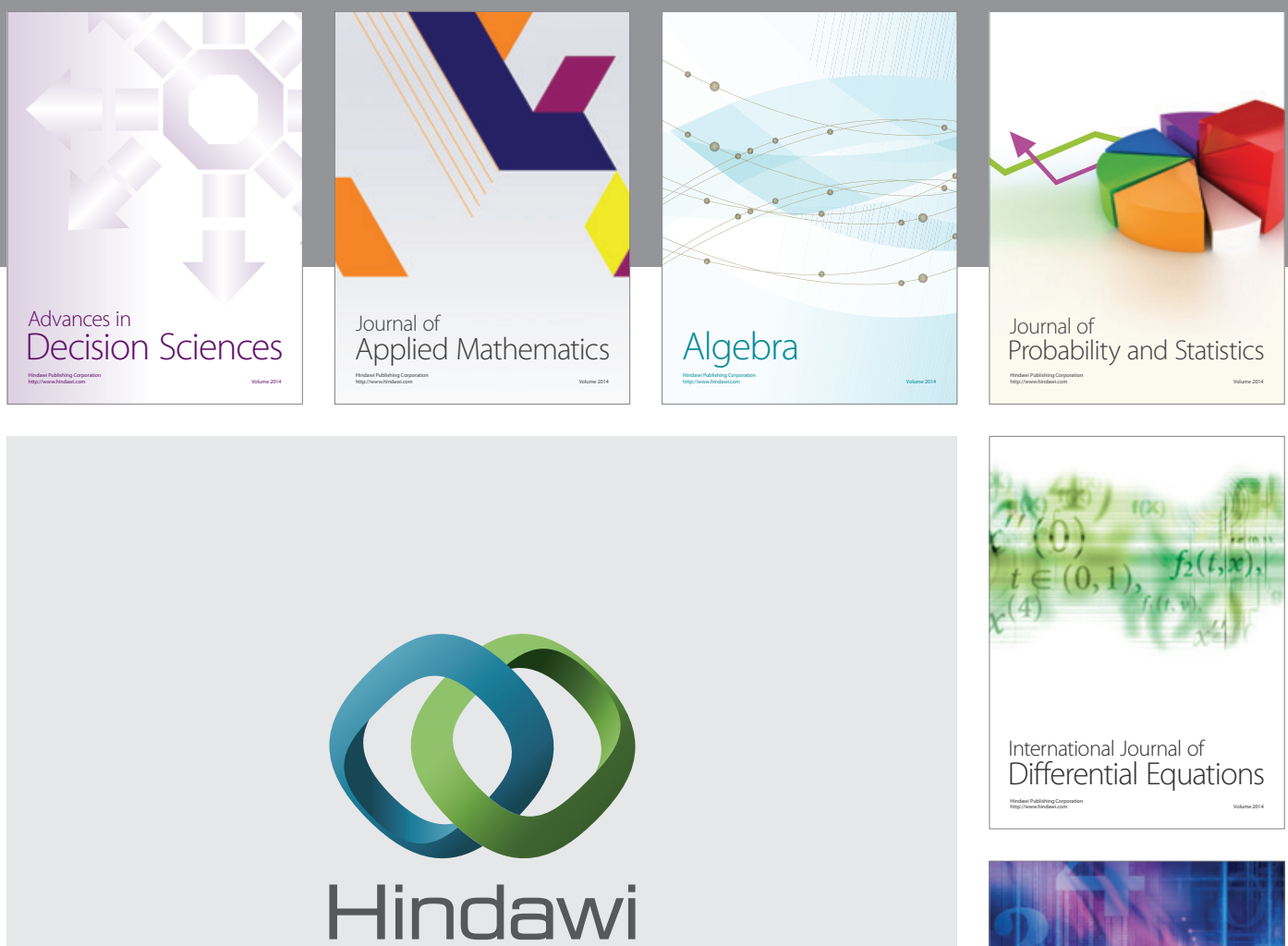

Submit your manuscripts at http://www.hindawi.com
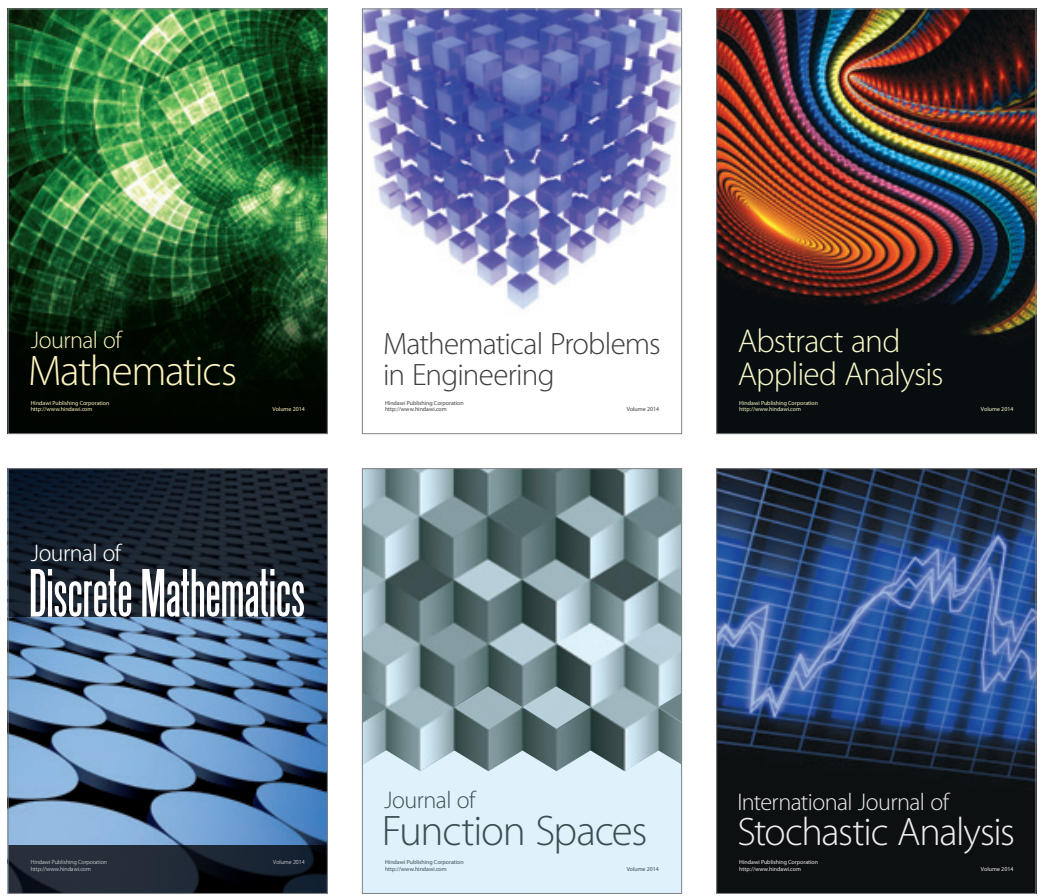

Journal of

Function Spaces

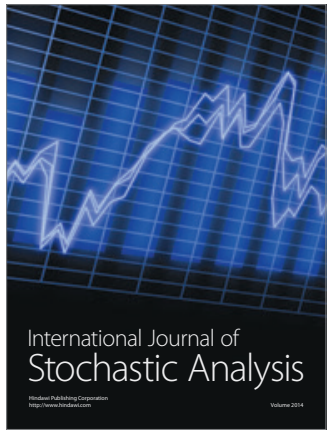

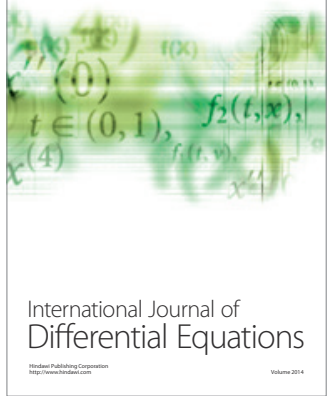
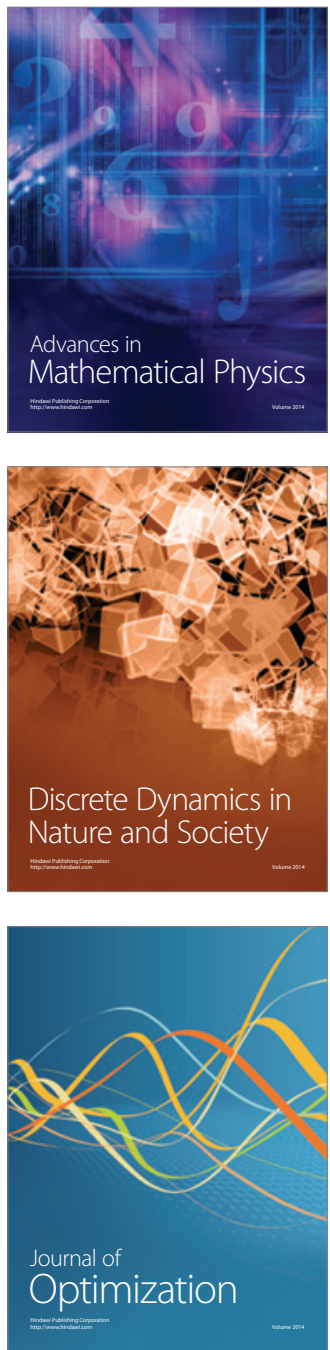